preferentially replicates in and causes the death of activated $\mathrm{CD} 4^{+} \mathrm{T}$ cells.

It is well known that chronic infecGetting to the bottom of $\mathrm{CD}^{+}$ T-cell loss

Previous studies of HIV pathogenesis have largely ignored events in the intestines of HIV-infected patients and have mostly concentrated on events in the blood, perhaps owing to the difficulty in obtaining intestinal lymphoid-tissue samples. However, two groups now report that the gastrointestinal tract has the most marked depletion of $\mathrm{CD}^{+} \mathrm{T}$ cells, and this occurs rapidly and at all stages of HIV infection, regardless of administration of highly active antiretroviral therapy (HAART).

Given that the gastrointestinal tract and other lymphoid tissues harbour most of the body's $\mathrm{CD} 4^{+}$ $\mathrm{T}$ cells and that, in the gut, a large number of these cells are activated and express the HIV co-receptor CC-chemokine receptor 5 (CCR5), the intestinal $\mathrm{CD} 4^{+} \mathrm{T}$ cells are potentially highly susceptible to infection with HIV. So, the authors of both studies set out to examine $\mathrm{CD} 4^{+}$ $\mathrm{T}$-cell depletion in the intestine of patients with HIV.

Danny Douek's group compared T-cell depletion in intestinal tissue, lymph nodes and blood from untreated HIV-infected patients (at all stages of disease) and from HIVuninfected individuals. As expected, the frequency of $\mathrm{CD} 4^{+} \mathrm{T}$ cells was significantly lower in each compartment in HIV-infected patients compared with HIV-uninfected individuals; however, the greatest loss of $\mathrm{CD}^{+}$ $\mathrm{T}$ cells was seen in the gastrointestinal tract, even in acute infection. This preferential depletion of intestinal $\mathrm{CD}^{+} \mathrm{T}$ cells was confirmed by histological studies, showing that lymphoid aggregates, which are abundant in normal intestinal-tissue samples, were largely absent in samples from patients with HIV. Further analysis revealed that the $\mathrm{CD} 4^{+} \mathrm{T}$-cell depletion in the gut was specific for those cells expressing CCR5 and the activation marker Ki67, which is consistent with the observation that HIV tion with HIV results in a state of general immune activation. Accordingly, Douek's group observed that effector memory $\mathrm{T}$ cells accumulated abnormally in the lymph nodes of HIVinfected patients. Moreover, this ated with collagen deposition in the lymph nodes, a marker of inflammation associated with chronic immune activation. This led the authors to suggest that disrupted lymphoid architecture might disturb normal lymphoid-tissue homeostasis, which might then impair $\mathrm{CD}^{+}$T-cell reconstitution of the gut following depletion by infection with HIV.

Martin Markowitz's group studied T-cell depletion in patients with acute or early HIV infection and compared untreated patients with those who had received HAART. Consistent with observations by Douek's group, in primary HIV infection, they found that $\mathrm{CD} 4^{+} \mathrm{T}$-cell depletion was most marked in the gastrointestinal tract and that it occurred before changes observed in the blood. Specifically, deletion mainly occurred in the effector sites (the lamina propria) as opposed to the inductive sites (Peyer's patches and lymphoid follicles) of the gut mucosa.

To examine whether HAART would allow the reconstitution of $\mathrm{CD}^{+} \mathrm{T}$ cells in the gut, they studied patients who had initiated HAART during primary infection. However, although $\mathrm{CD} 4^{+} \mathrm{T}$-cell numbers in the blood of these patients were mostly restored following HAART, reconstitution in the gastrointestinal tract remained incomplete despite up to 5 years of fully suppressive therapy.

Both of these studies highlight the need for further investigation of the mucosal compartment, given its crucial role in HIV infection, replication and persistence.

Lucy Bird

(2) References and links ORIGINAL RESEARCH PAPERS Brenchley, J. M. et al. $C D 4^{+} T$ cell depletion during all stages of HIV disease occurs predominantly in the gastrointestinal tract. J. Exp. Med. 200, 749-759 (2004) | Mehandru, S. et al. Primary HIV-1 infection is associated with preferential depletion of $\mathrm{CD} 4^{+}$ T lymphocytes from effector sites in the gastrointestinal tract. J. Exp. Med. 200, 761-770 (2004). increased T-cell activation was associ-

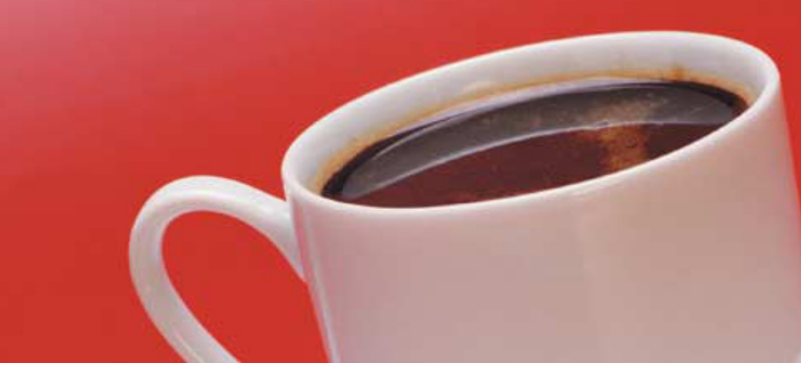

CYTOKINES

\section{Cytokine receptors take sugar}

Many proteins are modified in the endoplasmic reticulum through glycosylation of asparagine residues in a process known as $\mathrm{N}$-glycosylation. $\mathrm{N}$-glycans are further modified in the Golgi by enzymes, such as mannosidases and glycosyltransferases, to generate complex-type $\mathrm{N}$-glycans. New research published in Science now shows that mannoside acetylglucosaminyltransferase 5 (MGAT5) modifies cytokine-receptor N-glycans and that this regulates cytokine-mediated signalling.

MGAT5-mediated N-glycan modifications generate ligands for the galectin (GAL) family of lectins. It has previously been shown that GAL3 - which binds N-glycans modified by MGAT5 - can oligomerize and form a molecular lattice of galectins and glycoproteins at the cell surface and that GAL3 binding of MGAT5-modified N-linked glycans on the T-cell receptor opposes antigen-induced clustering.

Partridge et al. sought to investigate whether cytokine-receptor function was modulated by MGAT5 modification of N-linked glycans. MGAT5-deficient epithelial tumour cell lines derived from mammary tissue were less responsive to several cytokines, including epidermal growth factor (EGF) and transforming growth factor- $\beta$ (TGF- $\beta$ ), than MGAT5-sufficient cells, and the number of $\mathrm{N}$-glycans per cytokine receptor correlated with the decrease in sensitivity. The loss in EGF sensitivity was associated with a decrease in the level of cell-surface expression of the EGF receptor (EGFR), together with a decrease in the association of the EGFR with GAL3, a decrease in EGF-dependent activation of ERK (extracellular signal-regulated kinase) and an accumulation of the EGFR in the early endosomes. Similar data were obtained for the TGF- $\beta$ receptor II. Taken together, these data are consistent with the hypothesis that MGAT5-mediated modification of $\mathrm{N}$-linked glycans on these cytokine receptors allows the formation of molecular lattices that oppose receptor endocytosis.

The binding of TGF- $\beta$ to receptors on the surface of lipopolysaccharide-elicited peritoneal macrophages was decreased in the absence of MGAT5, as was TGF- $\beta$-dependent activation of SMAD2 and/or SMAD3. Furthermore, a functional role for MGAT5-mediated modification of N-glycans on cell-surface receptors was indicated by the observations that MGAT5-deficient macrophages were impaired in their ability to phagocytose latex beads and that leukocyte recruitment was delayed in both a model of skin inflammation and after intraperitoneal injection of thioglycollate.

This study shows that MGAT5-mediated modification of N-glycans on cytokine receptors regulates their cell-surface expression levels by balancing expression on the cell surface with loss through endocytosis and that this is an important mechanism controlling cellular cytokine responsiveness.

(1) References and links

Karen Honey

ORIGINAL RESEARCH PAPER Partridge, E. A. et al. Regulation of cytokine receptors

by Golgi N-glycan processing and endocytosis. Science 306, 120-124 (2004). 